\title{
Quiste de colédoco
}

\author{
Choledochal cyst \\ Juan Olmos Casas, ${ }^{*}$ Pedro Adrián González Zavala, \\ Jeffery Anzhony Cruz Robles, ${ }^{\S}$ Juan Enrique Olmos Sosall
}

\begin{abstract}
Palabras clave: Quiste de colédoco, hepaticoyeyunoanastomosis, conducto biliar, colangiocarcinoma, colangiopancreatografía retrograda endoscópica.

Keywords: Choledochal cyst, hepaticojejunostomy, bile duct, cholangiocarcinoma, endoscopic retrograde cholangiopancreatography.
\end{abstract}

${ }^{*}$ Cirujano General, Médico adscrito al Departamento de Cirugía General, Hospital General de Zona (HGZ) No. 36, IMSS Coatzacoalcos, Veracruz.

¥ Médico Interno de Pregrado adscrito al HGZ No. 36, IMSS Coatzacoalcos, Veracruz. $\S$ Médico Interno de Pregrado adscrito al Hospital General Regional No. 36, IMSS. Puebla, Puebla.

$\|$ Estudiante de primer año de Licenciatura en Medicina.

Universidad Popular Autónoma del Estado de Puebla (UPAEP). Puebla, Puebla.

Recibido: 05/03/2018 Aceptado: 26/03/2019

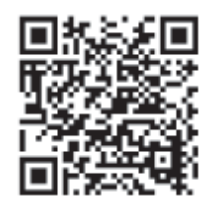

\section{RESUMEN}

Objetivo: Presentar el caso de un paciente con quiste de colédoco y su tratamiento, debido a su baja frecuencia y al riesgo elevado a desarrollar colangiocarcinoma. Presentación: mujer de 26 años con cuadro clínico de dolor abdominal en hipocondrio derecho y epigastrio, intolerancia a los colecistoquinéticos, además de ictericia conjuntival, acude a urgencias manejándose con analgésicos, se efectúa ultrasonido de hígado y vías biliares reportando vesícula normal, hígado normal, colédoco proximal de 7 $\mathrm{mm}$, colédoco distal dilatado de $35 \mathrm{~mm}$ con microlitos en su interior. Se ingresa a piso y se solicitan exámenes de laboratorio, los cuales se reportaron normales. Se efectúa colangiopancreatografía retrógrada endoscópica reportando quiste de colédoco fusiforme tipo I, por lo que se programa para cirugía efectuándole resección del quiste más colecistectomía y hepaticoyeyunoanastomosis en $\mathrm{Y}$ de Roux con prolene del 3-0. Se encuentra quiste de colédoco de $6 \times 4 \mathrm{~cm}$ que abarca desde el hepático común hasta páncreas. Evolucionó de manera satisfactoria, tolerando vía oral al cuarto día de operada, egresándose al séptimo día; es vista en la consulta con molestias propias de la cirugía, pero se encuentra anictérica y con herida quirúrgica bien cicatrizada, ultrasonido de control normal. Exámenes de laboratorio de control normales. Conclusiones: Es un padecimiento poco frecuente. Se encuentra un caso entre 100,000 a 150,000, de predominio en los países asiáticos; 20 a $30 \%$ se diagnostica en adultos. Tiene una incidencia elevada de desarrollar colangiocarcinoma, de ahí la importancia de la resección del quiste. El tipo I continúa siendo el más frecuente, reportándose así en la literatura mundial.

\section{ABSTRACT}

Objective: To present the case of a patient with choledochal cyst and its treatment, due to its low frequency and the high risk of developing cholangiocarcinoma. Presentation: A 26-year-old female with clinical signs of abdominal pain in the right hypochondrium and epigastrium, intolerance to cholecystokinetics, conjunctival jaundice, arrived to urgency with treatment analgesics, USG reporting normal vesicle, normal liver, proximal choledocholum $7 \mathrm{~mm}$, dilated distal common bile duct of $35 \mathrm{~mm}$ with microliths inside, are admitted to the floor requesting exams reporting $C B C, B C, T P$, normal TPT, normal LFT, ERCP is reported, and type I choledochal cyst is scheduled for surgical intervention With the resection of the choledochal cyst and cholecystectomy as well as Roux-en-Y hepaticojejunostomy with prolene 3-0, a $6 \times 4 \mathrm{~cm}$ choledochal cyst is found ranging from the common hepatic to pancreas, evolving satisfactorily, tolerating the oral route on the fourth day of surgery, leaving on the 7th day, is seen in the consultation, with discomfort of the surgery; being anicteric and with well-healed surgical wound, normal control USG. Control exams with normal CBC, BC, TP, TPT and LFT. Conclusions: It is an infrequent condition. One is found in 100,000 to 150,000 , predominantly in Asian countries 20 to $30 \%$ is diagnosed in adults. It has a high incidence of developing cholangiocarcinoma, hence the importance of cyst resection. Type I continues to be the most frequent, reporting in the world literature.

\section{INTRODUCCIÓN}

$\mathrm{E}^{\prime}$ quiste de colédoco es la malformación más frecuente de las vías biliares, tanto en niños como en adultos; dado que los límites entre el quiste de colédoco y ciertas anomalías morfológicas de la vía biliar de origen congénito no están hoy en día bien definidos, el término de dilatación congénita de la vía biliar principal parece preferible al de quiste de colédoco que es más restrictivo. Se define como entidad congénita ya que se ha encontrado en fetos y neonatos con el riesgo de desarrollar un colangiocarcinoma. Se presenta un caso cada

Citar como: Olmos CJ, González ZPA, Cruz RJA, Olmos SJE. Quiste de colédoco. Cir Gen. 2020; 42(1): 38-44. doi: $10.35366 / 92710$ 
100,000 a 150,000 nacimientos, cifras anglosajonas que se cree son comparables a las de México, y hasta uno en cada 1,000 en Japón y países asiáticos, 20 a 30\% se diagnostica en la edad adulta, en la mujer es tres a cuatro veces más frecuente. ${ }^{1-3}$ Fernández y colaboradores ${ }^{4}$ realizaron un estudio transversal, retrospectivo y descriptivo en el 2016, donde demostraron cuál es el porcentaje de la población mexicana más afectada, de un total de 24 pacientes que se registraron del 2006 al 2012, la población de estudio se tomó de los expedientes con diagnóstico de quiste de colédoco en los servicios de Gastroenterología y Cirugía Pediátrica del Hospital de Pediatría, Centro Médico Nacional Siglo XXI. ${ }^{4}$ De los 24 sujetos con expediente completo, 18 fueron mujeres $(75 \%)$, y seis hombres $(25 \%)$, siendo una relación 3:1. El grupo de edad más frecuente en el momento del diagnóstico de quiste de colédoco fueron los lactantes, en un $54.2 \%$; $20.8 \%$ en preescolares, $12.5 \%$ en escolares, $8.3 \%$ en neonatos y $4.2 \%$ en adolescentes. El grupo de edad de los pacientes en los que se realizó la cirugía con mayor frecuencia fue el de lactantes, en 15 pacientes, lo que corresponde a $62.5 \%$, seguido por cinco en la edad preescolar (20.8\%), y de forma similar en los escolares y adolescentes, con $4.2 \%$. Si tomamos en cuenta la edad de la cirugía, la presencia de eventos de colangitis se encontró con mayor frecuencia en lactantes $(81.8 \%)$, mientras que tanto en los preescolares como en los adolescentes, los eventos de colangitis no se presentaron. En la Ciudad de México $n=10$ o $41.7 \%$, Querétaro y Chiapas $\mathrm{n}=3$ o $12.5 \%$; el resto de los estados: Tlaxcala, Veracruz, Quintana Roo, Morelos, Guanajuato, Puebla, Guerrero y Aguascalientes refirieron un caso por estado.

En la actualidad, la preocupación es por la posibilidad de que evolucione a un colangiocarcinoma, ya que su frecuencia está aumentada hasta 20 veces en relación a la población sin esta patología; en cuanto al quiste tipo I su relación a malignidad va de un 2.5 a $26 \%$, y en comparación con los tipos II, III y IV su porcentaje de malignidad va de un $10-15 \%$, y riesgo de malignidad postquirúrgica $0.7 \%, 5,6$ lo mismo que las colangitis y las pancreatitis por reflujo de la bilis infectada o bacteriobilia. La transformación maligna es la complicación más grave, con un pronóstico de supervivencia muy pobre con resultados extremadamente desfavorables, con una supervivencia mediana informada de seis a 21 meses. $^{6}$

Su etiología sigue siendo tema de discusión, Hong-Tian Xia y su grupo ${ }^{7}$ en el 2015 demostraron que existen factores embriológicos y otros adquiridos, que dentro de los congénitos el más aceptado es la anormalidad en la unión pancreático biliar que van de 50 a $80 \%$, aunque no todas las causas se deben a una anormalidad anatómica. Con base en su estudio con 27 pacientes, se reveló que 21 presentaron reflejo pancreaticobiliar, y de estos 21, cuatro presentaron una unión anatómica pancreaticobiliar anormal, lo que ha demostrado que no sólo la anomalía anatómica de la unión biliopancreática es causa de quistes del colédoco, sino la presencia del reflujo pancreático biliar, que los niveles de amilasa pueden o no estar elevados y que la causa del reflujo pancreático biliar no está completamente explicada y lo único que explica la presencia de reflujo pancreaticobiliar (80\% de los pacientes anatómicamente normales) es la disfunción del esfínter de Oddi como causa primaria de quistes en el colédoco.

Fisiopatología del canal común y mala unión biliopancreática. La anomalía de la unión pancreatobiliar y el reflujo pancreatobiliar han cobrado mayor importancia en los últimos años, con el avance en el entendimiento de la fisiopatología y el aporte al cáncer de la vía biliar y la pancreatitis. La alteración de la unión pancreatobiliar se define como una anomalía congénita que consiste en la unión del conducto pancreático y vía biliar por fuera de la pared duodenal, formando un canal común y largo (> $15 \mathrm{~mm}$ ). De esta manera, el esfínter de Oddi no cumple su función y al existir una mayor presión hidrostática en el conducto pancreático, permite el reflujo a la vía biliar principalmente de jugo pancreático y de bilis; esto produce daño endotelial e hiperplasia y metaplasia epiteliales, que promueven la progresión a carcinoma. ${ }^{8}$

\section{CLASIFICACIÓN DE TODANI}

Tipo I: es la dilatación quística propiamente dicha del colédoco, casi global, que incluye el cístico; existen tres subtipos según la forma 
fusiforme o sacular, y el compromiso en el lugar de la convergencia.

I A: dilatación quística.

I B: dilatación focal.

I C: dilatación fusiforme del colédoco.

Tipo II: es el divertículo del colédoco y corresponde a dilataciones saculares y laterales, con cuello corto y estrecho; se encuentra en $2-10 \%$ de los pacientes.

Tipo III: corresponde al coledococele con dilatación de la porción terminal, que protruye en la luz duodenal y se presenta en $1.4-5 \%$ de los pacientes.

Tipo IV: la dilatación quística se puede asociar hasta en $20 \%$ de los pacientes con dilatación quística del cístico.

Se ha subdividido en tipo IVA cuando hay múltiples quistes intra y extrahepáticos, y IVB cuando son sólo extrahepáticos.

Tipo V: son dilataciones quísticas de localización exclusiva en la vía biliar intrahepática, llamada también enfermedad de Caroli, cuando se asocia a fibrosis periportal se denomina síndrome de Caroli (Figura 1). ${ }^{9}$

\section{COMPLICACIONES}

La morbilidad y mortalidad postoperatoria son típicamente muy bajas en los niños, mientras que las complicaciones postoperatorias se observan más comúnmente en los pacientes adultos. Las complicaciones tardías (más de

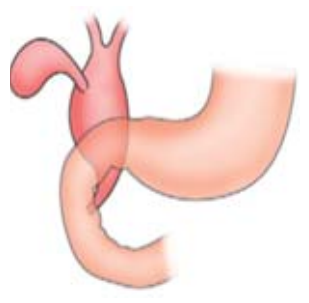

Tipo I C

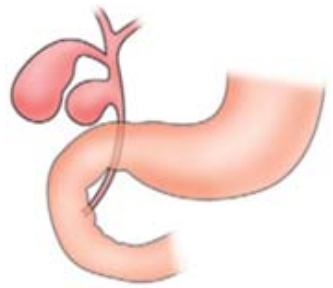

Tipo II

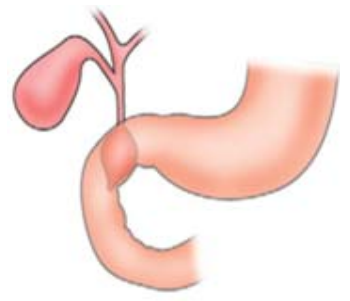

Tipo III

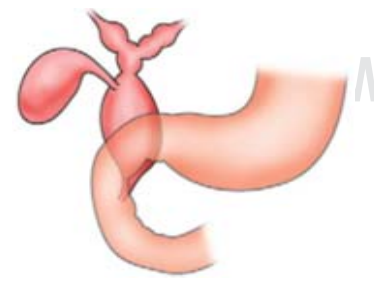

Tipo IV A

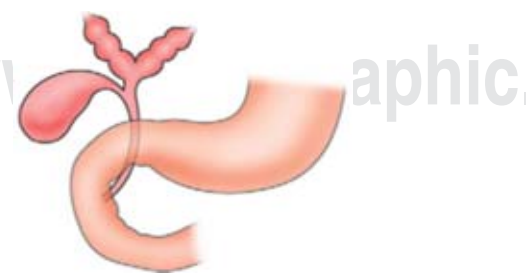

Tipo V
Figura 1: Clasificación de Todani.
30 días postoperatorias) ocurren en hasta $40 \%$ de los pacientes adultos e incluyen estenosis anastomótica, cáncer, colangitis y cirrosis. Dentro de los cuidados mediatos, se recomienda la vigilancia estrecha y consecutiva de los pacientes para intervenciones oportunas en el caso de ser necesario. ${ }^{10,11}$

\section{PRESENTACIÓN DEL CASO}

Mujer de 26 años que inicia padecimiento en junio del 2002 con dolor abdominal en epigastrio e hipocondrio derecho posterior a la ingesta de colecistoquinéticos, tratada entonces con paracetamol $500 \mathrm{mg}$ vía oral cada ocho horas y butilhioscina $10 \mathrm{mg}$ vía oral cada ocho horas en caso de dolor durante un mes, remitiendo de manera temporal el cuadro y presentando exacerbaciones durante su estancia en urgencias en sus primeras horas de hospitalización.

A la exploración física se mostraba con facies de dolor, ictericia conjuntival, campos pulmonares sin compromiso, abdomen blando, doloroso a la palpación de epigastrio e hipocondrio derecho, signo de Murphy, sin masas palpables, sin presencia de visceromegalias, sin datos de irritación peritoneal, extremidades íntegras, llenado capilar inmediato.

Se le realizan paraclínicos con el siguiente reporte: hemoglobina $13.4 \mathrm{mg} / \mathrm{dl}$, hematocrito $40.1 \%$, leucocitos $8.9 \times 10^{3} / \mathrm{l}$, plaquetas $234 \times$ $10^{9} / \mathrm{l}$, glucosa $76 \mathrm{mg} / \mathrm{dl}$ creatinina $0.8 \mathrm{mg} / \mathrm{dl}$, BUN $10 \mathrm{mg} / \mathrm{dl}$, bilirrubinas totales $4.1 \mathrm{mg} / \mathrm{dl}$, bilirrubina directa $3.6 \mathrm{mg} / \mathrm{dl}$, bilirrubina indirecta $0.5 \mathrm{mg} / \mathrm{dl}$, amilasa $75 \mathrm{U} / \mathrm{L}$, lipasa $95 \mathrm{UI} / \mathrm{L}$, AST $23 \mathrm{UI} / \mathrm{L}$, ALT $30 \mathrm{UI} / \mathrm{L}$, fosfatasa alcalina $100 \mathrm{U} / \mathrm{L}$, GGT $40 \mathrm{UI} / \mathrm{L}$, examen general orina sin alteraciones.

Se le realiza ultrasonido de hígado y vías biliares: hígado normal, colédoco proximal de $7 \mathrm{~mm}$, colédoco distal dilatado de $27 \mathrm{~mm}$ con microlitos en su interior, con aspecto en relación con quiste de colédoco, vesícula biliar de $82 \times 37 \mathrm{~mm}$ pared de $2 \mathrm{~mm}$ sin litos, páncreas, riñones y bazo normales.

Impresión diagnóstica (IDX) por ultrasonido: quiste de colédoco con microlitos en su interior (Figura 2).

Dada la indecisión de la paciente por aceptar estudios de extensión, manejo quirúrgico y mejoría con el tratamiento médico, se decide 

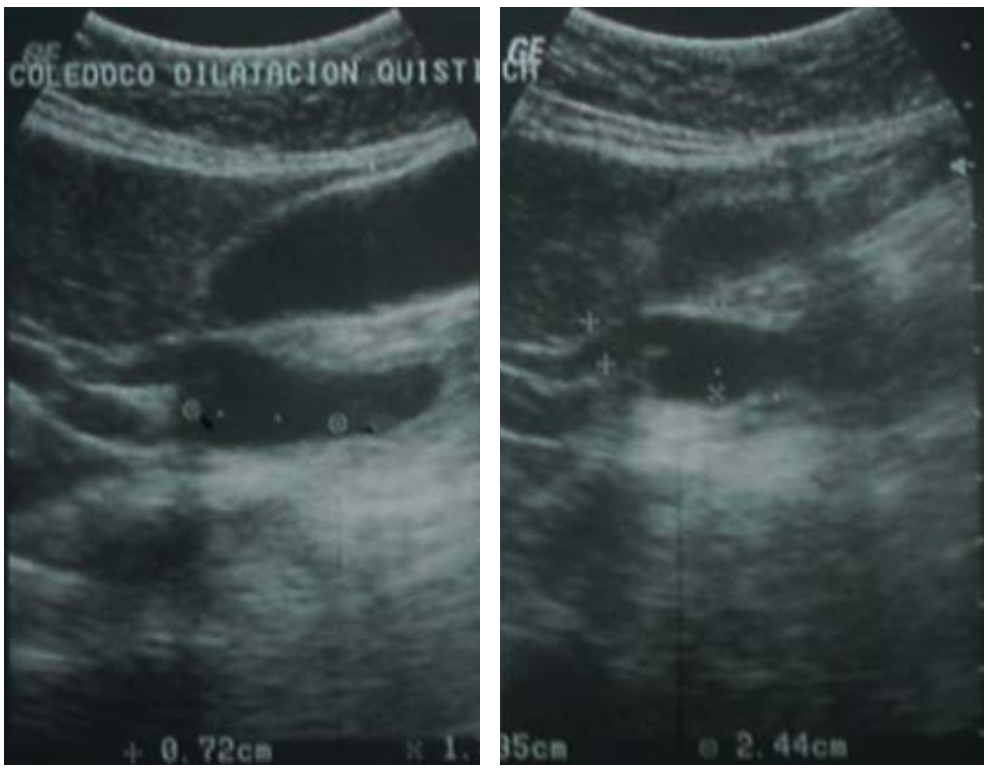

Figura 2: Se observa USG de hígado y vías biliares, con reporte de quiste de colédoco con microlitos en su interior.
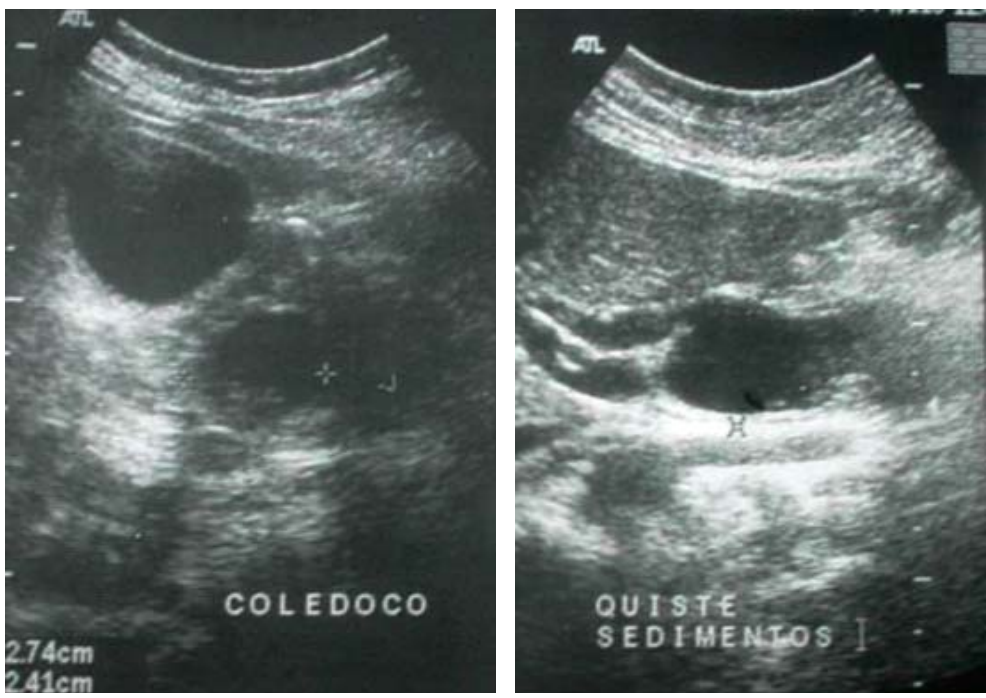

Figura 3: Se realiza otro estudio ultrasonográfico por la consulta externa, encontrándose hígado ecográficamente normal, vesícula biliar anecoica, mide aproximadamente $6.3 \times 3.1 \mathrm{~cm}$ pared de $4 \mathrm{~mm}$. Con presencia de quiste de colédoco en región distal con medidas $1.35 \times 2.4 \times 0.72 \mathrm{~cm}$.

dar el alta después de cuatro días de estancia hospitalaria y manejo por la consulta externa, solicitándole una colangiopancreatografía retrograda endoscópica (CPRE) y nuevo ultrasonido de hígado y vías biliares, así como exámenes de laboratorio, pruebas de funcionamiento hepático $(\mathrm{PFH})$, biometría hemática $(\mathrm{BH})$, química sanguínea (QS), tiempos de coagulación, con elevación de la bilirrubina total en $4.0 \mathrm{mg} /$ $\mathrm{dl}$ a expensas de la bilirrubina directa con 3.6 $\mathrm{mg} / \mathrm{dl}$, resto de resultados normales. Se ofrece manejo conservador a la paciente, e iniciar protocolo para programación quirúrgica del quiste de colédoco por la consulta externa, al no encontrarse urgencia quirúrgica.

Se realiza otro estudio ultrasonográfico por la consulta externa, encontrándose hígado ecográficamente normal, vesícula biliar anecoica en su interior mide aproximadamente $6.3 \times 3.1$ $\mathrm{cm}$, pared de $4 \mathrm{~mm}$. Con presencia de quiste de colédoco en región distal con medidas 1.35 x $2.4 \times 0.72 \mathrm{~cm}$ (Figura 3). Colédoco de calibre normal en su segmento proximal y diámetro de bordes delimitados sin compresión importante de órganos vecinos.

Previo consentimiento informado y con protocolo quirúrgico completo se efectúa colangiopancreatografía retrógrada endoscópica, por ser el recurso con el que cuenta nuestra institución, con el siguiente reporte.

Colangiopancreatografía retrógrada endoscópica: dilatación quística fusiforme de colédoco distal de $2.8 \mathrm{~cm}$ en su porción más ancha que inicia $1.6 \mathrm{~cm}$ por debajo de la confluencia de los hepáticos a la altura de la desembocadura del cístico, se extiende en una longitud de $4.8 \mathrm{~cm}$, en su extremo distal desemboca en un segmento de colédoco estrecho que resulta insuficiente para vaciar el quiste. La vesícula se opacificó parcialmente, el conducto hepático común, la Carina, hepáticos derecho e izquierdo tienen diámetro y configuración normal, la vía biliar intrahepática es normal (Figura 4).

Impresión diagnóstica: quiste de colédoco solitario fusiforme tipo I (clasificación de Todani). Ya cumplido el protocolo preoperatorio y definida la anatomía del quiste, así como su clasificación y relación con estructuras adyacentes, se lleva a cabo la cirugía electiva.

Bajo bloqueo mixto, previa asepsia y antisepsia de la región, se colocan campos estériles y se efectúa incisión subcostal derecha, disección por planos hasta llegar a cavidad abdominal; se identifica la vesícula biliar, se inicia disección del cístico y su arteria, posteriormente 

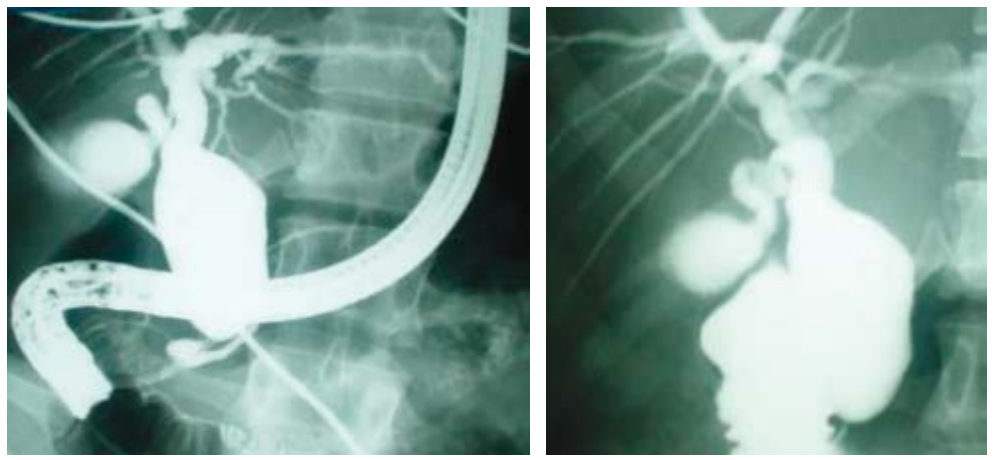

Figura 4: Dilatación quística fusiforme de colédoco distal de $2.8 \mathrm{~cm}$ en su porción más ancha, que inicia $1.6 \mathrm{~cm}$ por debajo de la confluencia de los hepáticos a la altura de la desembocadura del cístico, se extiende en una longitud de $4.8 \mathrm{~cm}$, en su extremo distal desemboca en un segmento de colédoco estrecho que resulta insuficiente para vaciar el quiste.

se diseca vesícula en forma anterógrada hasta llegar a su unión con colédoco, se diseca colédoco junto con el quiste, se efectúa pinzamiento, corte y ligadura de vasos sangrantes con seda del 2-0, posteriormente se secciona colédoco en su porción proximal y se diseca hasta llegar a su porción distal el cual se pinza, corta y liga, posteriormente se efectúa sección de yeyuno aproximadamente $40 \mathrm{~cm}$ del Treitz, se efectúa anastomosis terminolateral en dos planos con vicryl del 3-0 y seda del 2-0 a $70 \mathrm{~cm}$ del asa que va hacia el hepático común, posteriormente se efectúa hepatoyeyunoanastomosis con prolene 3-0 en un plano (Figura 5).

Hallazgos operatorios: quiste de colédoco de aproximadamente $6 \times 4 \mathrm{~cm}$, vesícula sin litos, hígado de aspecto normal (Figuras 6 y 7).
Evoluciona el postoperatorio de manera satisfactoria, iniciando vía oral al cuarto día y egresa al séptimo día de operada.

En la actualidad, se corrobora la excelente evolución y estado general, así como su reintegración a sus roles laborales y sociales mediante control por la consulta externa con USG y exámenes de laboratorio

\section{DISCUSIÓN}

Los quistes de colédoco se diagnostican en su mayoría en la infancia o adolescencia, y sólo 20-30\% ocurren en adultos. Es una patología muy rara en nuestro entorno, los síntomas más frecuentes son dolor abdominal en hipocondrio derecho e ictericia. Los adultos suelen presentar una clínica menos florida, lo que conlleva a un diagnóstico tardío. El abordaje terapéutico de los quistes biliares depende del tipo de quiste.

Dentro del protocolo para el manejo del quiste de colédoco, se debe clasificar mediante estudios de imagen, de los cuales la colangioRM es el de elección, al no contar con colangiorresonancia se utiliza CPRE en el presente caso, lo cual permite identificar la anatomía del quiste y planear el manejo quirúrgico, ${ }^{12}$ que consiste en resección completa del quiste más una hepatoenteroanastomosis en $Y$ de Roux, la cual en la actualidad se puede realizar por vía laparoscópica. ${ }^{13-15}$

En nuestro caso se realiza de manera satisfactoria colecistectomía y hepatoenteroanastomosis en $Y$ de Roux abierta, debido a que en la unidad no se cuenta con equipo ni experiencia en derivaciones biliodigestiva-laparoscópicas,
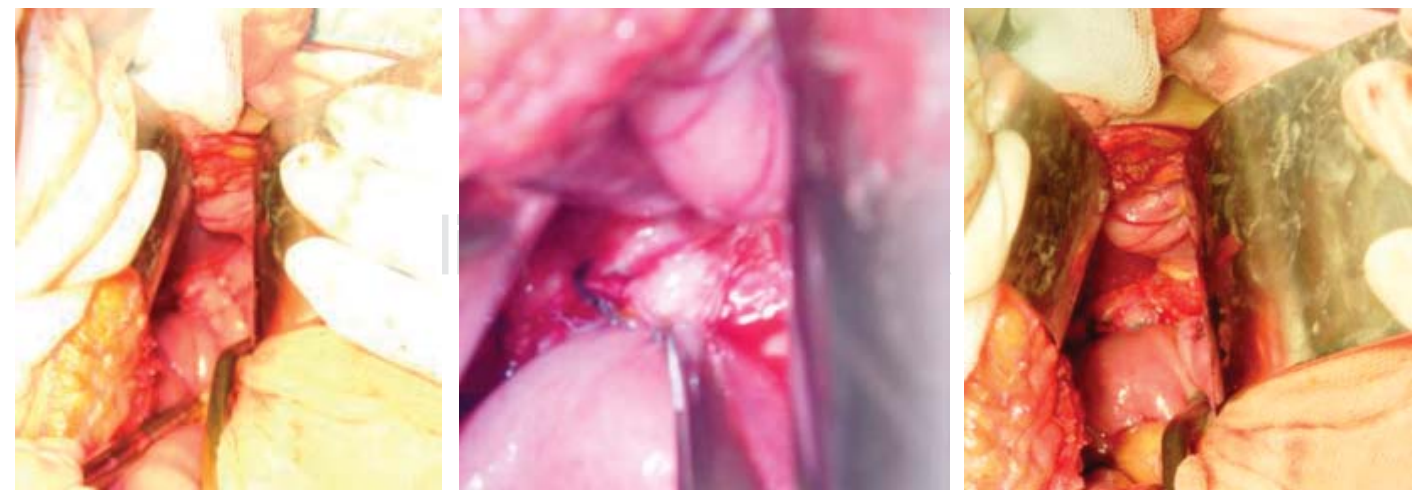

Figura 5: Hepatoenteroanastomosis en Y de Roux. Evolución del procedimiento. 


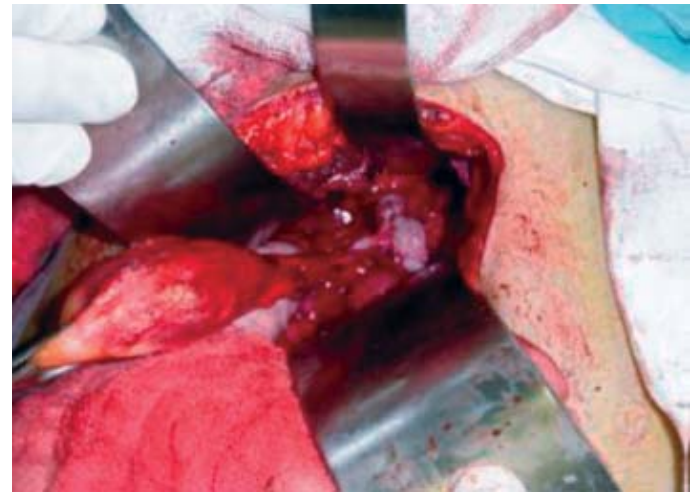

Figura 6: Extracción de vesícula y quiste de colédoco.

obteniendo resultados favorables con la paciente; se cita nuevamente al mes, seis meses y al año, con paraclínicos de control con pruebas de funcionamiento hepático, con valores dentro de parámetros normales. La persona continúa realizando sus actividades cotidianas.

El pronóstico para los pacientes con COlangiocarcinoma originados por un quiste en el colédoco es severo, tiene un reporte de supervivencia media de seis a 21 meses. El pobre pronóstico de pacientes con colangiocarcinoma se debe principalmente a que el tumor se diagnostica en etapas tardías; en los estudios realizados por Xiao-dong y colaboradores $^{7}$ fueron detectados pacientes con colangiocarcinoma en etapas tempranas, y ellos tuvieron un mejor pronóstico exactamente a

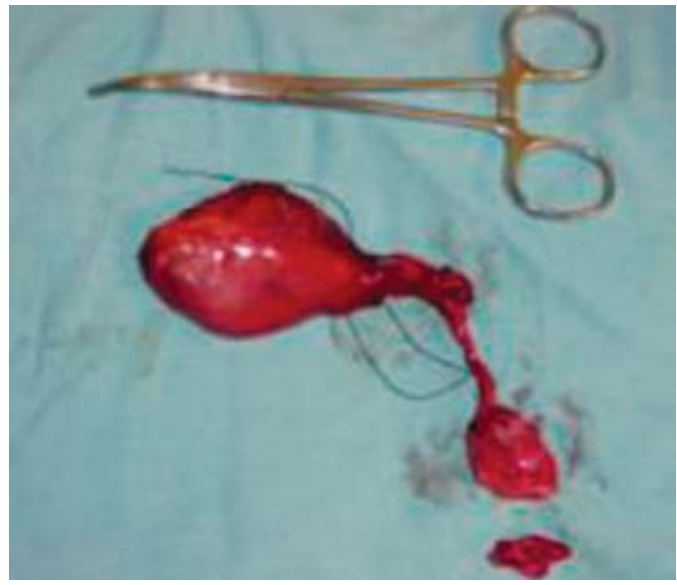

Figura 7: Quiste de colédoco de aproximadamente $6 \times 4$ $\mathrm{cm}$, vesícula sin litos, hígado de aspecto normal.

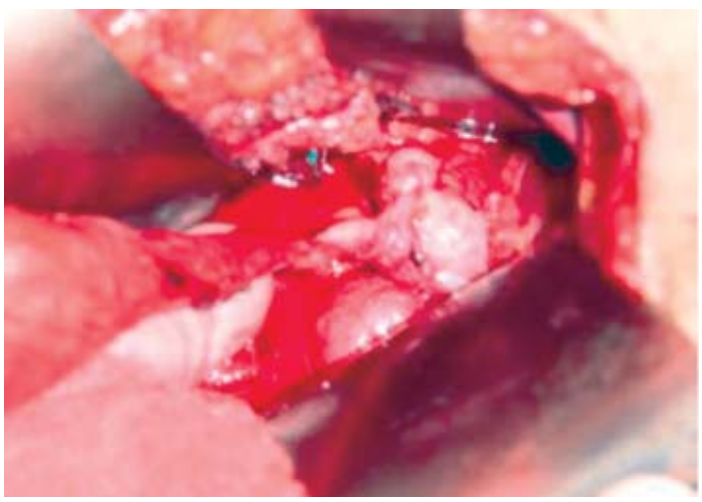

cinco años en pacientes con estadios la, Ib y Ila del colangiocarcinoma, alcanzando 90.4, 40.0 y $25.15 \%$ de supervivencia respectivamente.

\section{CONCLUSIÓN}

En general, la resección QC tiene un pronóstico excelente, con un $89 \%$ sin eventos y un total de cinco años. El pronóstico para el paciente con colangiocarcinoma que surge de un quiste de colédoco es sombrío, con una supervivencia media notificada de seis a 21 meses. El mal pronóstico de los pacientes con colangiocarcinoma se atribuyó principalmente a la etapa tardía de los tumores en el momento del diagnóstico. La malignidad rara vez se observó en pacientes con quistes extirpados en la infancia, pero el riesgo de carcinogénesis aumenta con la edad. El riesgo de transformación maligna era inferior a 1\% si el quiste coledocal aparecía antes de los 10 años, pero aumentó a 14\% por encima de los 20 años.

\section{REFERENCIAS}

1. Alvarado-García R, Reza-Villa A, Uribe-Ramos D, Gallego-Grijalva JE. Quiste gigante de colédoco. Informe de un caso pediátrico. Cir Ciruj. 2002; 70: 40-43.

2. Acevedo-Polakovich D, Rivas-Rivera IA, Rodríguez-López A. Quiste de colédoco: cuadro clínico, diagnóstico y tratamiento. Acta Med. 2015; 13: 171-176.

3. Xia HT, Wang J, Yang T, Liang B, Zeng JP, Dong JH. Sphincter of Oddi dysfunction and the formation of adult choledochal cyst following cholecystectomy: a retrospective cohort study. Medicine (Baltimore). 2015; 94: e2088. doi: 10.1097/MD.0000000000002088.

4. Fernández-Bobadilla N, Flores-Calderón J, BernabéGarcía M, Lagarda-Cuevas J. Caracterización clínica de 
niños con quiste de colédoco. An Med (Mex). 2016; 61: 11-19.

5. Fernández de Córdova L, Valverde-Guerrero G, Narváez-Ludeña GG, Orellana-Cobos DF, Vélez AP. Quiste de colédoco, reporte de caso en el Hospital "Vicente Corral Moscoso". Rev Méd Hosp José Carrasco Arteaga. 2014; 6: 180-183.

6. Jiménez-Ballester MA, Soria-Aledo V, MartínLorenzo JG, Olalla-Muñoz JR, Giménez-Bascuñana A, Alcaraz-Mateos E, et al. Quiste de colédoco tipo II intrapancreático del adulto malignizado: duodenopancreatectomía. Rev Esp Enferm Dig. 2014; 106: 223-226.

7. Xiao-dong H, Wang L, Liu W, Liu Q, Qu Q, Bing-lu L, et al. The risk of carcinogenesis in congenital choledochal cyst patients: an analysis of 214 cases. Ann Hepatol. 2014; 13: 819-826.

8. Baca PE, Peralta A, Argüello BJ, Castañeda OAB, Hernández AM, Ochoa GP. Tratamiento laparoscópico del quiste de colédoco en niños. Acta Med. 2015; 13 : 38-41.

9. de Vries JS, de Vries S, Aronson DC, Bosman DK, Rauws EA, Bosma A, et al. Choledochal cysts: age of presentation, symptoms, and late complications related to Todani's classification. J Pediatr Surg. 2002; 37: 1568-1573.

10. Chaurand-Lara M, Canto-Cervera A. Quistes de colédoco. Evid Med Invest Salud. 2015; 8: 37-40.

11. Soares KC, Arnaoutakis DJ, Kamel I, Rastegar N, Anders R, Maithel S, et al. Choledochal cysts: presentation, clinical differentiation, and management. J Am Coll Surg. 2014; 219: 1167-1180.

12. Vivas-Colmenares GV, Millán-López A, De Agustín AJC. Optimización en la estrategia diagnóstica y terapéutica en el quiste de colédoco. Rev Chil Cir. 2015; 67: 278284.

13. Giha S, Redondo Y, Quintero G. Quiste de colédoco: diagnóstico y manejo intraoperatorio. Pediatr. 2016; 49: 64-67.
14. Gadelhak N, Shehta A, Hamed H. Diagnosis and management of choledochal cyst: 20 years of single center experience. World J Gastroenterol. 2014; 20: 7061-7066.

15. Crisanto-Campos BA, Torres-Ruíz MF, Vázquez-Bailón IJ, Dávila-Zenteno MR, Arce-Liévano E, CárdenasLailson LE, et al. Quiste de colédoco en adultos: resección totalmente laparoscópica. Reporte de caso. Rev Mex Cir Endoscop. 2013; 14: 38-42.

Consideraciones y responsabilidad ética: Privacidad de los datos. De acuerdo a los protocolos establecidos en el centro de trabajo de los autores, estos declaran que han seguido los protocolos sobre la privacidad de datos de pacientes preservado su anonimato. El consentimiento informado del paciente referido en el artículo se encuentra en poder del autor.

Financiamiento: No se recibió apoyo financiero para la realización de este trabajo.

Conflicto de intereses: Los autores declaran que no existe ningún conflicto de intereses en la realización del trabajo.

Correspondencia:

Dr. Juan Olmos Casas

Departamento de Cirugía General,

Hospital General de Zona No. 36,

IMSS, Coatzacoalcos, Ver.

Nuevo León Núm. 521,

Col. Petrolera, 96500,

Coatzacoalcos, Veracruz.

Tel: 9211158050

E-mail: dr.olmoscasas@hotmail.com 